\title{
Polymer Optical Fiber-based Magnetic Sensor Based on Magneto-optical Effect
}

\author{
Yao-Nan Wang, ${ }^{1}$ Hsing-Hui Huang, ${ }^{1}$ Li-Wen Chen, ${ }^{1}$ \\ Wei-Hua Lu, ${ }^{2}$ Kun-Huang Wang, ${ }^{1}$ and Yung-Chuan Chen ${ }^{1 *}$ \\ ${ }^{1}$ Department of Vehicle Engineering, National Pingtung University of Science and Technology, \\ Pingtung 91201, Taiwan \\ ${ }^{2}$ Department of Material Engineering, National Pingtung University of Science and Technology, \\ Pingtung 91201, Taiwan
}

(Received May 18, 2018; accepted March 20, 2019)

Keywords: plastic optical fiber, magnetic microspheres, magnetic sensor

A magnetic sensor comprising two polymer optical fibers (POFs) and a capillary tube filled with a solution of magnetic microspheres is presented. In the proposed device, the ray path through the capillary tube changes as a result of the variation in microsphere distribution caused by the presence of a magnetic field. The corresponding change in the voltage output signal of the avalanche photodiode (APD) is then used to derive the intensity of the magnetic field. Experimental results show that the proposed sensor has a sensitivity of $6.2 \mathrm{mV} / \mathrm{G}$ for magnetic field intensities in the range of 30-100 G. From the experimental results, an empirical expression is formulated to relate the magnetic field intensity with the output voltage ratio. The magnetic field intensities predicted using this formulation deviate from the measured values by less than $1 \%$.

\section{Introduction}

In many technical applications, e.g., structural health monitoring, position measurements, and so forth, it is necessary to measure the variation in the intensity of a magnetic field with a high degree of accuracy. In many cases, the magnetic field can be measured directly using traditional devices such as a Gauss meter. However, for harsh or difficult-to-access environments, fiber optic sensors provide a more convenient and reliable approach since they provide a higher resistance toward vibration, electromagnetic interference, and corrosion. ${ }^{(1)}$ Compared with glass optical fibers sensing applications, polymer optical fibers (POFs) have the advantages of a large core diameter, ductility, light weight, and ease of connection. ${ }^{(2-4)}$ Consequently, the power attenuation characteristics of POFs and their feasibility for sensing applications have attracted increasing attention in recent reports. ${ }^{(5-7)}$

Radojevic et al. proposed a magnetic field sensor based on an optical fiber with a composite magnetic coating comprising polyethylene-co-vinyl acetate (EVA) and SmCo5 magnetic powder. ${ }^{(1)}$ Their results showed that the sensor had a high sensitivity to a magnetic field and the *Corresponding author: e-mail: chuan@mail.npust.edu.tw https://doi.org/10.18494/SAM.2019.2277 
reversibility of a signal. Rashleigh constructed a fiber optic magnetic field sensor comprising a single-mode fiber bonded to a nickel cylinder. ${ }^{(8)}$ It was shown that the device had a sensitivity of $1.76 \times 10^{-2} \mathrm{rad} / \mathrm{m}$ Oe permitting the detection of magnetic fields with an intensity of as low as the fiber intensity of $4.4 \times 10^{-6} \mathrm{Oe} / \mathrm{m}$. Davino et al. presented a magnetic field sensor for hysteresis compensation consisting of a magneto-elastic rod and a fiber Bragg grating (FBG) strain sensor. $^{(9)}$ Their experimental results showed that the sensor had a sufficient accuracy for the detection of magnetic fields. Quintero et al. presented an optical sensor for DC and AC magnetic field measurements comprising an FBG sensor embedded within an optical fiber coated with a thick magnetostrictive composite layer consisting of Terfenol-D particles dispersed in a polymeric matrix. ${ }^{(10)}$ It was shown that the sensor had a resolution of $0.4 \mathrm{mT}$ with no preload or $0.3 \mathrm{mT}$ under a compressive prestress of 8.6 MPa. Orr and Niewczas proposed an optical fiber sensor system for the simultaneous measurements of the magnetic field strength and temperature based on the use of FBGs with low linear birefringence as multiplexable transducers. ${ }^{(11)}$ Djinovic et al. presented a fiber optic magnetic field sensor for structural health monitoring applications in which the intensity of the magnetic field was detected by measuring the change in the length of the sensing wire. ${ }^{(12)}$ Their results showed that the sensor had a sensitivity of approximately $50 \mathrm{~nm}$ for magnetic field intensities in the range of 0-800 $\mu \mathrm{T}$. Dai et al. proposed an optical magnetic field sensor consisting of a capillary tube etched with an FBG sensing element and filled with $\mathrm{Fe}_{3} \mathrm{O}_{4}$ magnetic fluid. ${ }^{(13)}$ Their experimental results showed that the sensitivity of the proposed device improved as the diameter of the FBG decreased.

In this study, we propose a relatively simple technique based on a magnetic sensor incorporating two POFs and a capillary tube filled with a solution of $8 \mu \mathrm{m}$ magnetic microspheres diluted in water. Accordingly, we perform a series of experimental tests to evaluate the avalanche photodiode (APD) output voltage in the proposed sensor for magnetic field intensities ranging from 0 to $130 \mathrm{G}$. In addition, experimental results are used to construct an empirical model for relating the voltage output ratio of the sensor with the magnetic field intensity.

\section{Experimental Setup}

Figure 1 presents a schematic illustration of the experimental setup used to evaluate the performance of the proposed POF-based magnetic sensor. Figure 1(a) shows the main parts of the equipment, namely, a light source (Photom, Model 205A, Tokyo, Japan), a Gauss meter (FW BELL 5180, USA), a DC power supply (Instek GPS-2303, Taiwan), an APD (APD module C5460-01, Hamamatsu, Japan), a shielded connector block with signal-labeled data acquisition (DAQ, BNC-2110, National Instruments, Austin, TX), and a computer. In the experimental setup for magnetic field measurement, the light from the light source $(660 \mathrm{~nm}$, Photom, Model 205A, Japan) was launched into the sensor from one POF, and the transmission light intensity corresponding to the variation in magnetic field intensity was measured at the other POF with an APD. The high-gain amplification APD converts the corresponding transmission light intensity to the electricity signal received and recorded with DAQ (BNC-2110) and the 


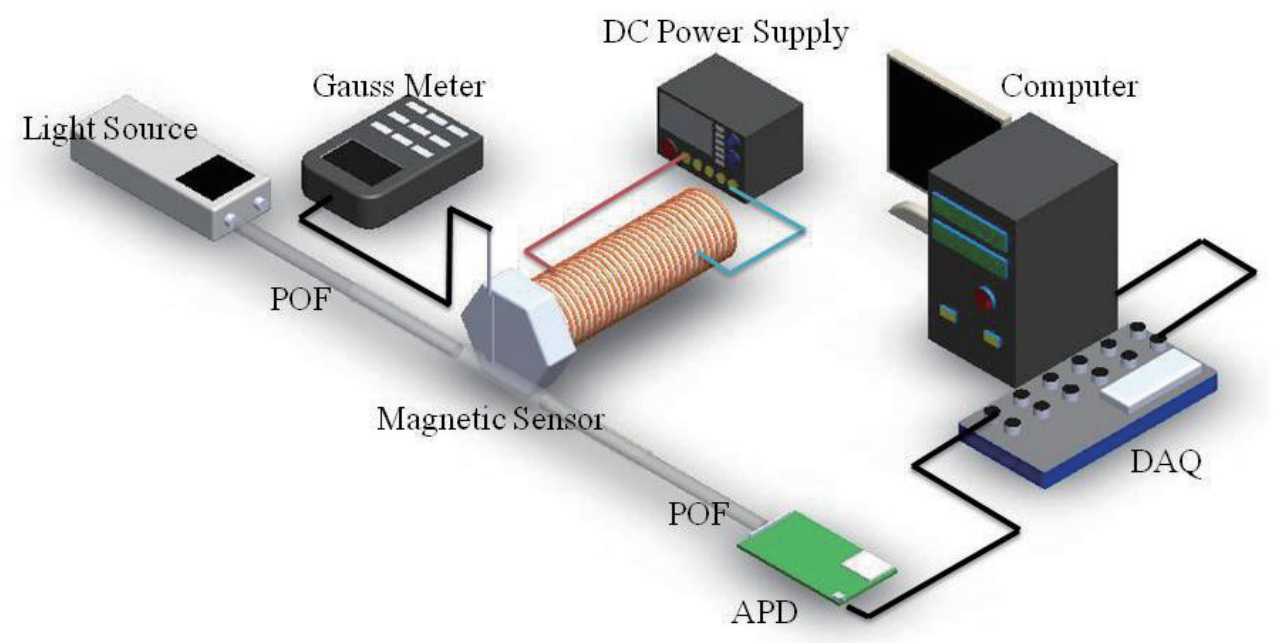

(a)

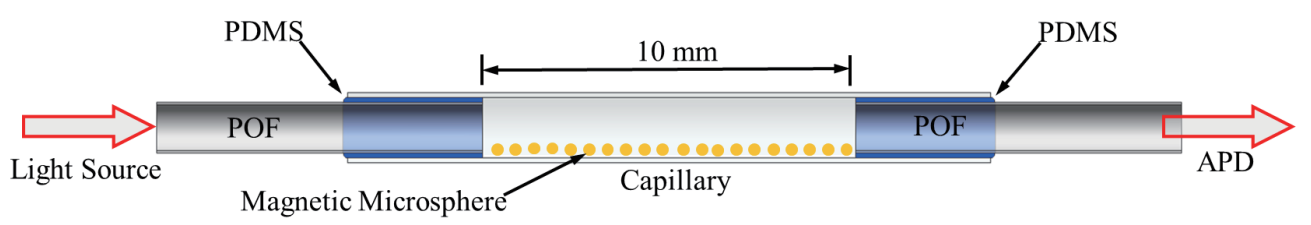

(b)

Fig. 1. (Color online) (a) Experimental setup used to measure output voltage and (b) components and dimensions of magnetic sensor.

computer. Figure 1(b) shows the components and dimensions of the proposed magnetic sensor that comprises two lengths of step-index-type SH-4001 POF (Mitsubishi Rayon Company Ltd., Japan, length: $500 \mathrm{~mm}$ ) and a glass capillary tube (Assistant, Germany, inner diameter: $1.1 \mathrm{~mm}$, length: $75 \mathrm{~mm}$ ). The POF has a core diameter of $0.98 \mathrm{~mm}$, a cladding diameter of $1 \mathrm{~mm}$, a coating diameter of $2.2 \mathrm{~mm}$, and a numerical aperture (NA) of 0.5. Moreover, the core and cladding are fabricated from polymethyl methacrylate (PMMA) and fluorinated polymer with refractive indices of $n_{c o}=1.492$ and $n_{c l}=1.402$, respectively.

In constructing the sensor, $10 \mu \mathrm{l}$ of magnetic fluid comprising magnetic microspheres ( $8 \mu \mathrm{m}$, density: $1.1-1.2 \mathrm{~g} / \mathrm{cm}^{3}$, UMC 4F, Bangs Laboratories, Fishers, IN, USA) diluted 20 times with de-ionized (DI) water and $0.01 \%$ Tween 20 was added to prevent magnetic microsphere agglomeration. The magnetic fluid was injected into the capillary tube, which was then sealed at both ends by means of polydimethylsiloxane (PDMS), resulting in the confinement of magnetic fluid particles at a length of approximately $10 \mathrm{~mm}$ at the midpoint of the capillary tube [see Fig. 1(b)]. The ends of the POFs were polished manually and then pushed over the capillary tube and adjusted such that they slightly overlapped with the two ends of the plug within the tube. The free end of the POF on the left of the capillary tube was connected to the light source (a light-emitting diode with a wavelength of $660 \mathrm{~nm}$ ), while that of the POF on the right of the tube was connected to the APD. The APD was connected to the DAQ, which was connected 
in turn to a PC. During the experiments, APD output signals were collected and analyzed by means of a self-written Lab View program. As shown in Fig. 1, the magnetic source had the form of a steel bolt wrapped with a current-carrying coil. The intensity of the magnetic field was adjusted by increasing or decreasing the current as required and measured at the midpoint of the sensor using the Gauss meter probe.

\section{Results and Discussion}

Figure 2 shows the variation in APD output signal voltage over time in the absence of a magnetic field. The voltage is observed to be negative since the APD module voltage signal has a negative photoelectric coefficient. For example, the signal indicates a voltage drop when the light enters the APD module. The average value (voltage) of these variations is denoted as $V_{0}$, i.e., the initial voltage of the sensor obtained when no magnetic field is applied. The average output signal is denoted as $V$ a obtained when a magnetic field is applied. Moreover, upon inspection, the mean output voltage $\left(V_{0}\right)$ is equal to approximately $-3.984 \mathrm{~V}$.

Figure 3 presents the numerical simulation results for the magnetic flux density of the bolt. The magnetic flux density is about $1.923 \times 10^{-3} \mathrm{~T}$ (Tesla). It is seen that the magnetic flux density at the surface of the bolt is inhomogeneous from the simulation results. Notably, the magnetic flux density in the middle is higher than those at both ends. The magnetic microspheres are expected to spread widely in the capillary owing to the inhomogeneous magnetic flux density that reduces the light intensity significantly and increases the magnetic sensor sensitivity. Figures 4(a) and 4(b) show the distributions of the magnetic microspheres within the capillary tube in the absence and presence of a magnetic field, respectively. When no magnetic field is applied, the microspheres sink toward the bottom of the capillary tube under the effects of gravity. However, when a magnetic field is applied, the microspheres are attracted toward the upper-right side of the tube, as shown in Fig. 4(b). Notably, the number of magnetic microspheres attracted toward the magnet depends on the intensity of the applied

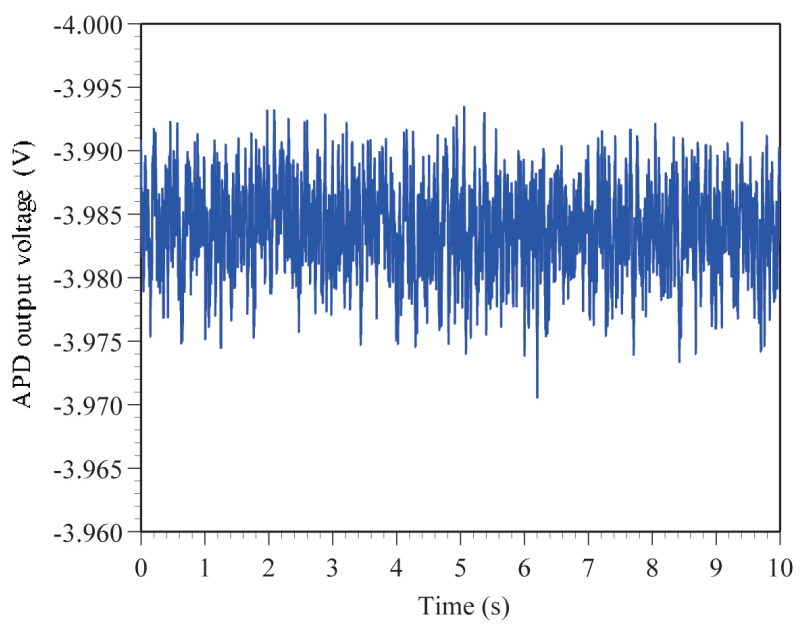

Fig. 2. (Color online) Variation in APD output signal voltage in the absence of magnetic field. 

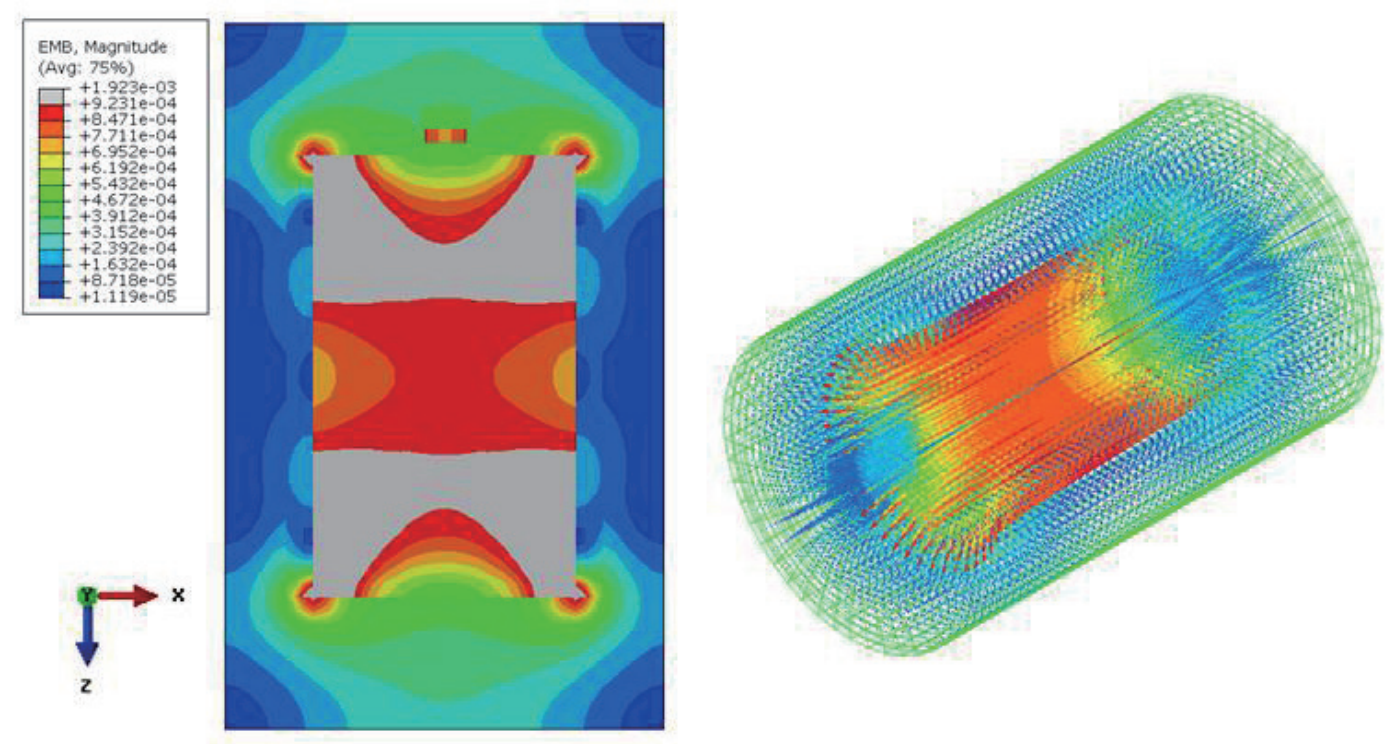

Fig. 3. (Color online) Numerical simulation results for the magnetic flux density of the bolt.

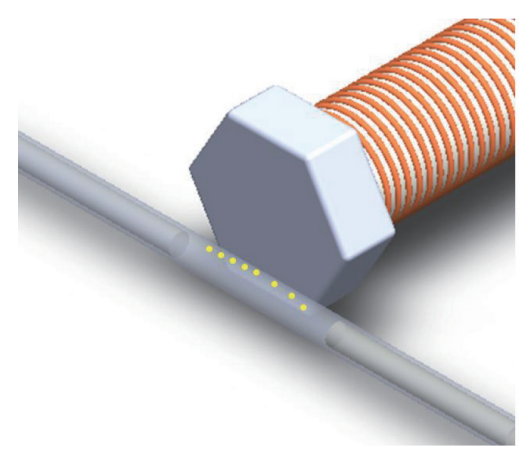

(a)

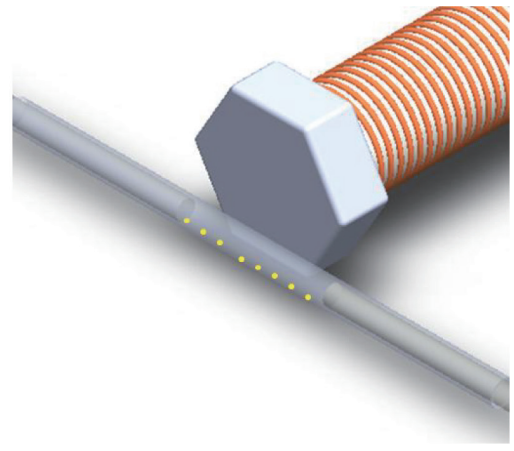

(b)

Fig. 4. (Color online) Distributions of magnetic microspheres in the (a) absence and (b) presence of magnetic field.

magnetic field. The change in the distribution of the microspheres affects the ray path through the capillary tube and therefore prompts a variation in APD output signal voltage from which the magnetic field intensity can be inversely derived. The density of magnetic microspheres is slightly higher than that of DI water and the magnetic sensor response time is short, but the magnetic microsphere settlement time is long.

Figure 5 shows the average APD output voltage with the magnetic field intensity for three repeated experiments using the same POF sensor. The corresponding magnetic field intensity is monitored using a commercial Gauss meter simultaneously. The variation in APD output signal voltage is indicated by the size of the error bars and the maximum deviation is about $\pm 60 \mathrm{mV}$ at 30 Gauss. For a given magnetic field intensity, the voltage varies by approximately $4 \%$. This is considered to be due to the high sensitivity of the APD toward changes in detected light intensity. In general, the results show that for magnetic field intensities in the range of $0-30 \mathrm{G}$, 


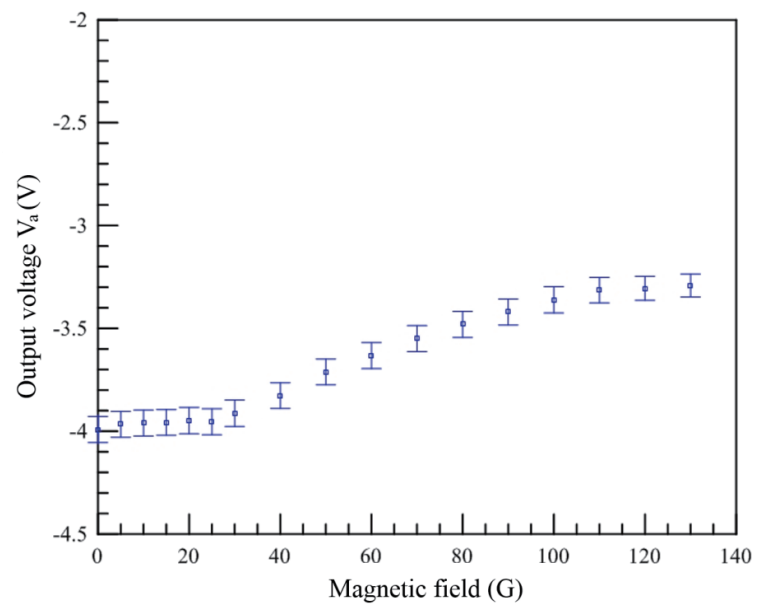

Fig. 5. (Color online) Variation in voltage with magnetic field intensity.

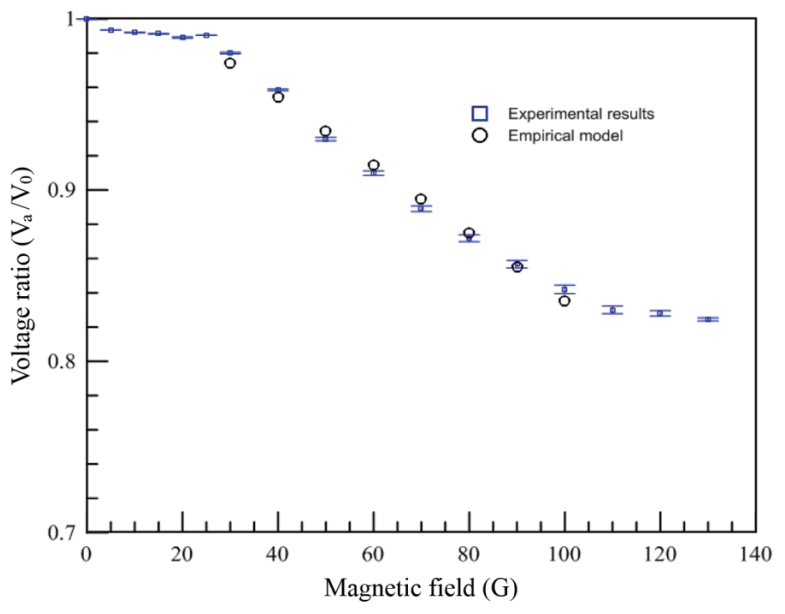

Fig. 6. (Color online) Variation in normalized voltage with magnetic field intensities in range of $30-$ $100 \mathrm{G}$.

the voltage remains approximately constant. In other words, the magnetic field has no effect on the microsphere distribution within the capillary tube at low intensities. However, the voltage increases linearly as the magnetic field intensity increases to $100 \mathrm{G}$. As the intensity increases further to $140 \mathrm{G}$, the voltage remains approximately constant once again owing to the saturation of the magnetic microspheres in the upper-right region of the capillary tube [see Fig. 4(b)]. In general, the results confirm that the output voltage of the APD varies as a function of the number of microspheres attracted toward the magnet. In addition, the results indicate that the proposed sensor has an effective working range of 30-100 G. Upon inspection, the sensitivity of the sensor in this working range is found to be $6.2 \mathrm{mV} / \mathrm{G}$.

Figure 6 shows the variation in voltage drop ratio $\left(V_{a} / V_{0}\right)$ with the intensity of the applied magnetic field over three repeated experiments. Note that $\mathrm{V}$ is the APD output voltage measured in the presence of a magnetic field and $V_{a} / V_{0}$ is the mean output voltage obtained in the absence of a magnetic field (see Fig. 2). Notably, the use of a normalized voltage ratio allows the effects of variations in the initial voltage $V_{0}$ to be ignored. By applying a curve fitting technique to the experimental data presented in Fig. 6 for magnetic field intensities ranging from 30 to $100 \mathrm{G}$, the voltage ratio and magnetic field intensity are shown to be related as

$$
\frac{V_{a}}{V_{0}}=-1.989 \times 10^{-3} \mathrm{G}+1.034
$$

The maximum deviation between the results obtained from this equation for the magnetic field intensity and the experimental results is found to be less than $1 \%$. 


\section{Conclusions}

In this study, a POF-based sensor comprising a capillary tube filled with magnetic microspheres suspended in DI water for measuring the variation in magnetic field intensity is proposed. Experimental results have shown that the sensor has a sensitivity of $6.2 \mathrm{mV} / \mathrm{G}$ over the working range of 30-100 G. Moreover, it has been shown that the voltage output ratio of the sensor and the magnetic field intensity are related as Eq. (1). The magnetic field intensities predicted using this formulation deviate from the measured values by less than $1 \%$. Compared with the existing optical fiber magnetic field sensor, optical transmission signals are recorded and analyzed utilizing an optical spectrum analyzer (wavelength shift or transmission loss). The magnetic field sensor proposed in this study utilizes an APD to measure the light intensity variation caused by the effect of a magnetic field with simple fabrication processes that provides a compact, simple, low-cost, and accurate tool for magnetic field intensity measurement.

\section{Acknowledgments}

The authors gratefully acknowledge the financial support provided to this study by the Ministry of Science and Technology, R.O.C., under Grant No. MOST 103-2221-E-020-015.

\section{References}

1 V. Radojevic, D. Nedeljkovic, N. Talijan, D. Trifunovic, and R. Aleksic: J. Magn. Magn. Mater. 272-276 (2004) e1755.

2 J. Zheng, X. Dong, P. Zu, L. Y. Shao, C. C. Chan, Y. Cui, and P. P. Shum: Opt. Express 21 (2013) 17863.

3 Z. Tong, P. Luan, Y. Cao, W. Zhang, and L. Li: Opt. Eng. 54 (2015) 087106.

4 G. H. Su, J. Shi, D. G. Xu, H. W. Zhang, W. Xu, Y. Y. Wang, J. C. Feng, and J. Q. Yao: IEEE Sens. J. 16 (2016) 8489.

5 J. H. Kuang, P. C. Chen, and Y. C. Chen: Sensors 10 (2010) 10198.

6 Y. C. Chen, L. W. Chen, and W. H. Lu: Sensors 11 (2011) 8741.

7 W. H. Lu, L. W. Chen, and Y. C. Chen: Sensors 12 (2012) 7485.

8 S. C. Rashleigh: Opt. Lett. 6 (1981) 19.

9 D. Davino, C. Visone, C. Ambrosino, S. Campopiano, A. Cusano, and A. Cutolo: Sens. Actuators, A 147 (2008) 127.

10 S. M. M. Quintero, A. M. B. Braga, H. I. Weber, A. C. Bruno, and J. F. D. F. Araújo: Sensors 10 (2010) 8119.

11 P. Orr and P. Niewczas: Sens. Actuators, A 163 (2010) 68.

12 Z. Djinovic, M. Tomic, and C. Gamauf: Procedia Eng. 5 (2010) 1103.

13 J. Dai, M. Yang, X. Li, H. Liu, and X. Tong: Opt. Fiber Technol. 17 (2011) 210.

\section{About the Authors}

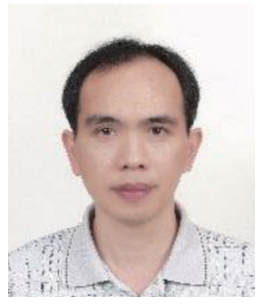

Yao-Nan Wang received his M.S. and Ph.D. degrees from National Cheng Kung University, Taiwan, R.O.C., in 2002, and 2008, respectively. From 2009 to 2012, he was an assistant professor at National Pingtung University of Science and Technology, Taiwan. Since 2017, he has been a professor at National Pingtung University of Science and Technology, Taiwan. His research interests are in MEMS, biomedical engineering, and sensors. 


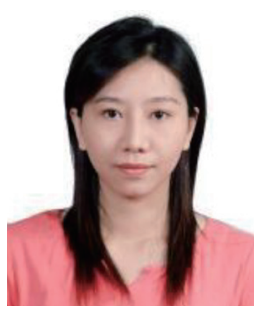

Hsing-Hui Huang received her B.S. degree from National Chen Kun University, Taiwan, in 1997 and her M.S. and Ph.D. degrees also from National Chen Kun University, Taiwan, R.O.C., in 1999 and 2004, respectively. She is an associate professor at National Pingtung University of Science and Technology, Taiwan. Her research interests are in mechanical design, chassis design, and sensors.

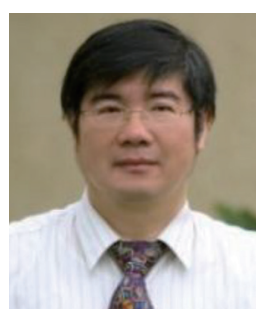

Li-Wen Chen received his B.S. and M.S. degrees from National Tsing Hua University, Taiwan, R.O.C., in 1985 and 1987, respectively, his second M.S. degree from the University of Southern California, USA, in 1989, and his Ph.D. degree from the University of California at Berkeley, USA, in 1995. From 1996 to 2008, he was an associate professor at National Pingtung University of Science and Technology, Taiwan. Since 2008, he has been a professor at National Pingtung University of Science and Technology, Taiwan. His research interests are in sensors, mechatronics, and controller design.

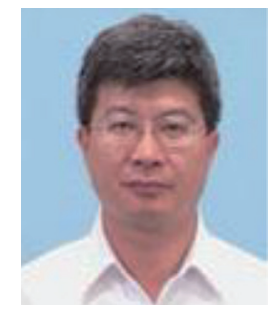

Wei-Hua Lu received his B.S. degree from Tunghai University, Taiwan, R.O.C., in 1988, and his M.S. and Ph.D. degrees from National Sun Yatsen University, Taiwan, R.O.C., in 1990 and 1995, respectively. From 2003 to 2007, he was an assistant professor at National Pingtung University of Science and Technology, Taiwan. Since 2012, he has been a professor at National Pingtung University of Science and Technology, Taiwan. His research interests are in polymer material, advanced IC assembly technology, structural stress analysis, and sensors.

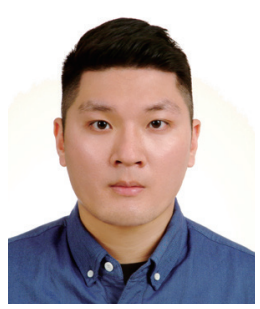

Kun-Huang Wang received his B.S. and M.S. degrees from National Pingtung University of Science and Technology, Taiwan, R.O.C., in 2011 and 2013, respectively. He is currently an engineer at Taiwan Semiconductor Manufacturing Company Limited. His research interests are in polymer optical fiber sensors.

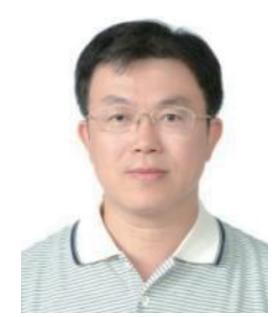

Yung-Chuan Chen received his M.S. and Ph.D. degrees from National Sun Yat-sen University, Taiwan, R.O.C., in 1991 and 1996, respectively. From 2000 to 2004, he was an assistant professor at National Pingtung University of Science and Technology, Taiwan. Since 2007, he has been a professor at National Pingtung University of Science and Technology, Taiwan. His research interests are in deformed polymer optical fibers, thermal and contact stress analysis, and sensors. 